EOMmUn: Communication et organisation

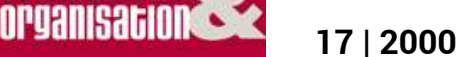

Europe et communication

\title{
Analyse de la démarche Éthique en gestion
}

\section{Philippe Saielli}

\section{OpenEdition}

Journals

Édition électronique

URL : http://journals.openedition.org/communicationorganisation/2378

DOI : 10.4000/communicationorganisation.2378

ISSN : 1775-3546

\section{Éditeur}

Presses universitaires de Bordeaux

\section{Édition imprimée}

Date de publication : 1 mai 2000

ISSN : 1168-5549

\section{Référence électronique}

Philippe Saielli, "Analyse de la démarche Éthique en gestion », Communication et organisation [En ligne], 17 | 2000, mis en ligne le 27 mars 2012, consulté le 30 avril 2019. URL : http:// journals.openedition.org/communicationorganisation/2378; DOI : 10.4000/ communicationorganisation. 2378

Ce document a été généré automatiquement le 30 avril 2019.

(c) Presses universitaires de Bordeaux 


\title{
Analyse de la démarche Éthique en gestion
}

\author{
Philippe Saielli
}

1 Lorsqu'on s'intéresse à l'éthique des affaires, on constate que cette notion est étudiée de deux manières distinctes ${ }^{1}$ : soit par l'intermédiaire de la formalisation d'une éthique organisationnelle, soit l'accent est mis sur le processus réflexif qui préside aux choix; cela concerne le manager confronté à des arbitrages complexes. Cet article a pour objectif de proposer un état des lieux des fondements et des limites de la démarche éthique dans un contexte organisationnel. La littérature sur le sujet accorde une place importante à la délibération. Par délibération, on entend la démarche éthique mise en œuvre par le manager intégrant une réflexion sur les valeurs et les enjeux de la situation. Par manager, on désigne une personne en position d'autorité dont les décisions ont des conséquences directes sur d'autres personnes (collaborateurs, clients, etc.).

2 Concernant cette question de la démarche éthique en entreprise, il est intéressant de s'interroger sur les éléments suivants :

- Quels sont les fondements d'un appel à la réflexion morale dans un contexte d'entreprise dont la finalité essentielle est une finalité économique?

4 - La forme de la démarche éthique ne varie-t-elle pas en fonction des référentiels moraux qui la sous-tendent?

5 - N'existe-t-il pas un risque de promouvoir la démarche éthique en survalorisant la rationalité de l'acteur? La rationalité sous-tendant la délibération serait-t-elle la même que celle du mode de pensée technico-économique?

6 C'est en fonction de ce questionnement que nous nous proposons de rendre compte de la littérature. Cet article a une orientation volontairement gestionnaire, puisque nous nous sommes intéressés uniquement aux auteurs qui abordent la question de l'éthique en entreprise. 


\section{La démarche éthique sous l'angle de la délibération}

7 Parler de démarche éthique, c'est introduire des questionnements liés aux conflits et aux ajustements de valeurs dans la pratique du manager. Plus précisément, on parle de délibération pour désigner la volonté d'ordonnancer et d'ajuster les multiples valeurs qui sous-tendent l'agir et les conséquences proches ou lointaines du choix de l'action. Elle se présente comme une instance dynamique, un processus rationnel, permettant aux managers de prendre en compte la dimension éthique de leur pratique.

Selon Bergmann ${ }^{2}$, « respecter une certaine éthique en affaires revient à attribuer des valeurs aux diverses conséquences des activités économiques et à établir des priorités entre elles. Ainsi comprise, l'éthique en gestion ne consiste pas en un canon mais en un processus : celui d'une réflexion continue sur les conséquences multiples des actions ».

La délibération s'affirme dans la volonté d'élaborer une décision dans la distanciation. On demande au responsable de prendre du recul par rapport aux événements, de hiérarchiser les valeurs en jeu dans une situation donnée et de mesurer les conséquences de l'action à entreprendre. Cela conduit à localiser le souci éthique au niveau de la responsabilité de l'acteur.

La délibération en tant que processus réflexif ne vaut que s'il existe des convictions qui permettent une hiérarchisation et une pondération des valeurs. En ce sens la responsabilité est inséparable d'une certaine idée du bien à promouvoir ${ }^{3}$. Certains discours mettent en avant une représentation homogène de l'organisation. Il s'agit des discours affichant une homogénéité des valeurs au sein de l'entreprise; par exemple, l'idée selon laquelle en travaillant pour les objectifs de l'entreprise, l'individu travaille à son propre développement. Il est important de souligner que l'idée même de délibération est légitimée par deux éléments qui vont à l'encontre de cette homogénéisation: la liberté de l'acteur et la multitude des référentiels axiologiques au sein de l'entreprise.

\section{La reconnaissance de la liberté du décideur}

10 Dans les situations de complet déterminisme, le manager dispose d'une possibilité d'opter pour un choix d'action parmi d'autres. Que l'individu le veuille ou non, il est libre. Certes, il n'est pas libre de choisir ce qui lui arrive, mais il peut réagir de telle ou telle façon aux événements.

11 La délibération s'exprime dans l'utilisation que chacun fait de sa liberté. Elle apparaitt comme le moyen pour le manager d'assumer cette liberté. Par cette réflexion, il prend en compte la dimension éthique de sa pratique. Cette reconnaissance de la liberté du manager est à mettre en parallèle avec l'idée qu'aucune procédure, convention ou règle ne dispensera la personne de faire un choix. Thévenet et Bibard ${ }^{4}$ expliquent ceci par le fait, que tous les repères dans l'entreprise qui donnent une orientation et une signification à l'action ne répondent jamais totalement au besoin de sens de l'acteur.

C'est en raison de cette liberté de choix qui est constitutive de l'être humain que la réflexion sur les valeurs à promouvoir prend son sens. On reconnaît ainsi au manager la capacité à développer une logique d'action qui lui est spécifique. En fonction de leur historicité et du contexte, les acteurs produisent leur propre finalité et idéal à suivre. Ce 
qui veut dire que le manager construit ses choix éthiques en s'appropriant les valeurs du contexte. Il donne ainsi un sens à son agir.

\section{La reconnaissance de la pluralité des valeurs dans le champ de l'entreprise.}

Reconnaître la pluralité des valeurs implique d'accepter la relativité des différents codes moraux. En effet, la délibération signifie qu'il y a une position à prendre, un choix à assumer qui affecte d'autres personnes, dans un contexte où aucune valeur ne s'impose d'elle-même. Souvent, il faut décider entre des valeurs positives différentes et parfois contradictoires, par exemple les valeurs du travail bien fait, de la réussite de l'entreprise, du respect de la justice vis-à-vis de membres de l'entreprise, du respect des procédures légales. En effet, il n'est plus aujourd'hui possible de penser la décision des managers dans une logique de mono rationalité. c'est ce que montre la littérature anglo-saxonne en se référant à la notion d'éthique post-moderne ${ }^{5}$. On reconnaît ainsi la multiplicité des rationalités en présence dans chaque décision. Le choix d'une rationalité particulière ne correspond pas à un choix irrationnel, mais pré-rationnel.

On peut saisir cette multiplicité de valeurs présentes dans l'entreprise en considérant, à l'instar de Louart ${ }^{6}$, que les aspects technico-économiques et les aspects axiologiques sont intimement liés dans l'action. Dans tout processus de gestion cohabitent une rationalité technico-économique, issue des objectifs économiques, des techniques, des outils et des procédures qui leur sont associés et une rationalité socio-politique qui se réfère aux intérêts et aux enjeux des différents acteurs engagés dans cette situation?

C'est parce qu'il y a une mise en valeurs de l'action quotidienne par un travail réflexif des managers que l'appel à la délibération acquiert sa légitimité. Le sens de l'agir a besoin d'être questionné à la fois par rapport à une évaluation des situations concrètes et par rapport à une idée plus globale du juste et de l'injuste.

16 L'autre idée, c'est que la délibération sous-entend une certaine maturation de la personne. En effet, lorsque l'on reconnaît au manager une liberté de choix, on lui attribue aussi une capacité à se distancier des décisions immédiates et des réactions spontanées face à une situation. À l'aide de la notion de délibération, on demande aux gestionnaires de gérer cette liberté par une analyse rationnelle des enjeux et des conséquences de l'action. On fait ici référence à la notion de volonté. Seule la personne est capable de définir le sens qu'elle souhaite donner à sa vie. Mais cela ne veut pas dire qu'elle peut faire n'importe quoi. En agissant sans limites, elle pourrait provoquer des effets néfastes sur le fonctionnement de la communauté dans laquelle elle travaille.

À ce propos, Perrot ${ }^{8}$ insiste sur l'importance d'un retour sur soi pour toute prise de décision. Selon lui, aucune procédure ne dispensera le manager de faire un choix. Les conventions ou les règles sont toujours limitées, à la fois, par rapport à la complexité des situations et par rapport à des valeurs morales plus universelles.

Perrot montre aussi que choisir l'un ou l'autre des repères qui déterminent le bien agir, constitue toujours une adhésion de la conscience à un choix parmi d'autres. En cela, la décision est relative et implique de bien comprendre la signification de tel ou tel repère. Si on peut formuler les questions éthiques, soit en référence aux valeurs traditionnelles (responsabilité, justice, solidarité), soit en référence à des solutions plus contemporaines (régulation par le marché, administration, négociation), aucune de ces formulations ne 
fournira la réponse a priori. Seul le décideur peut la donner. Dans l'illustration qui suit, nous détaillons les différents repères structurels à la délibération et leurs significations sous-jacentes. doivent guider la réflexion éthique des dirigeants. Ces principes sont religieux ou laïques, leurs messages résident dans l'affirmation de la dignité de l'homme. C'est d'ailleurs dans ce sens qu'Orsoni ${ }^{9}$ définit la délibération: «L'exercice de la morale reste un effort de discernement dans les actes, dans les décisions des gestionnaires - du bien et du mal. Car, en définitive, qui ne pourra jamais prouver qu'il y a deux éthiques : une pour les individus et une autre pour les entreprises? Qui pourra démontrer que la fin et les moyens ne sont pas de la même nature ? Le problème moral de l'entreprise est celui de l'homme, et le problème pour le gestionnaire consiste à chercher, non pas le Bien absolu, mais à chaque fois, pas à pas un bien pour l'homme ». 
Quelle soit à l'intérieur ou à l'extérieur du domaine d'action qu'est l'entreprise, l'éthique pose la question du bien agir. Elle appelle au respect de la valeur humaine, c'est-à-dire à l'acceptation de l'autre à la fois comme un autre soi-même et dans sa différence. La mise en œuvre pratique, dans les décisions de gestion quotidiennes, de ce respect accordé à la dignité humaine requiert un processus de questionnement continu sur le sens de l'action à mettre œuvre.

Dans cette perspective, les auteurs ne distinguent pas l'éthique individuelle de l'éthique des affaires. C'est la même problématique : celle de la reconnaissance mutuelle ${ }^{10}$. Cela veut dire qu'on ne peut pas en gestion, se cantonner à une quête de vérité et d'objectivité (comme le sous-tend la logique technico-économique). Il faut aussi rechercher le bien dans une logique d'humanisation des rapports à l'autre (individuels ou collectifs). Pour y parvenir, il est nécessaire de délibérer sur les fins et les moyens. Certes, il ne s'agit pas dans cette logique de nier les contraintes de la gestion d'une entreprise, mais comme le souligne Orsoni, rien n'interdit la délibération, « car la volonté de la fin est bien ce qui donne un sens aux moyens. $»^{11}$

Les auteurs d'obédience religieuse abordent aussi l'éthique des affaires de cette manière. Pour Moussé, Falise, Regnier ${ }^{12}$ et Perrot, c'est le gestionnaire, qui par sa volonté d'intégrer une réflexion prudente dans sa pratique professionnelle, introduit un éclairage à partir de valeurs universelles.

Par exemple, Falise et Regnier définissent la démarche éthique ainsi : «Ce n'est d'aucune façon une morale ou un code venus de l'extérieur et qui imposeraient de façon prédéterminée, la solution moralement acceptable, mais plutôt une démarche, une façon de penser et d'agir qui prenne en compte l'ensemble des faits, des enjeux et des valeurs, qui assume et gère les tensions dans une dynamique de progrès qui soit attentive à l'écoute des autres."

Quel que soit le champ d'application de l'éthique, ces auteurs rappellent que cette notion est avant tout une ouverture à l'universel. C'est au manager d'assurer le lien entre les contingences de la situation et des principes moraux plus globaux. Pour assurer cette liaison le manager a besoin de fonder un cadre éthique solide et d'accepter le risque qu'encourt celui qui s'engage dans l'action. Il n'existe pas de décision parfaite. Tout choix est partiel et hasardeux. Toute décision tient une part d'aléatoire. Cependant il importe que le manager intègre chacune de ses expériences comme une base d'apprentissage de ses propres valeurs et reste ouvert à une compréhension toujours plus fine de l'essence même des valeurs universelles.

33 La démarche éthique est un processus de questionnement et de discernement qui demande à rester ouvert au débat. En gestion des ressources humaines, Louart ${ }^{13}$ développe cette approche. Il montre que le manager est de fait limité dans sa connaissance de la réalité. Ses modèles d'action seront toujours restreints par rapport à la complexité du réel. Pour dépasser ce non-savoir (l'aporie logique à laquelle il est confronté) dans l'expérience de l'action, le responsable a besoin de développer un cadre éthique. Il se fixe ainsi des limites normatives à ses actions fondées sur l'honnêteté du langage et le respect d'autrui comme un autre soi-même. Ce cadre doit également tenir compte des contraintes organisationnelles. La délibération prend alors la forme d'un débat à la fois intra-psychique et intersubjectif.

Dans une seconde approche, l'éthique des affaires est pensée dans sa spécificité. Certes, elle n'est pas déconnectée des universaux moraux. Mais elle est avant tout définie comme 
la formalisation d'un système de règles. Ce dernier est associé à un ensemble de valeurs qui propose ce qui est bien ou mal dans un domaine d'action particulier. Jarislowsky ${ }^{14}$ et Gélinier ${ }^{15}$ partagent cette approche. Mais l'ouvrage le plus représentatif de cette conception est celui est coordonné par de la Bruslerie ${ }^{16}$.

C'est donc par l'établissement de codes de déontologie, qui se référent à des valeurs universelles, que l'on suscite chez le gestionnaire une activité réflexive. Ces règlements sont spécifiques au domaine d'action d'une profession ou de la gestion d'une organisation. Ils comprennent généralement un arsenal de sanctions en cas de non respect des règles. Ces codes ont pour fonction d'obliger les gestionnaires à respecter un minimum d'éthique.

On trouve ici l'idée que tous les managers n'ont pas la même capacité ni le même intérêt à intégrer une démarche éthique. C'est pourquoi, il est essentiel de traduire les valeurs universelles par des règles et des procédures concrètes permettant d'assurer un minimum de déontologie dans l'entreprise ou dans une profession.

\section{La délibération en référence aux valeurs contingentes.}

37 Pour certains, la délibération ne doit (et ne peut) pas prendre en compte des valeurs universelles généralement inspirées de la philosophie morale. Car ces valeurs sont souvent trop déconnectées de la réalité du monde de la gestion ${ }^{17}$.

Pour d'autres, l'éthique des affaires résulte des pressions exercées par l'environnement de l'entreprise. Nantel et Weeks ${ }^{18}$ illustrent cette façon de voir: "Ainsi, la notion de développement durable, qui fonde les pratiques d'un nombre croissant d'entreprises, est en bonne partie le fruit des pressions qu'exercent les consommateurs. Un tel constat force inévitablement les gestionnaires de marketing, s'ils veulent être cohérents avec l'orientation de leur discipline à considérer sérieusement l'adoption d'une approche déontologique ».

39 La délibération des hommes du marketing doit prendre en compte les attentes sociales. C'est l'étude de l'opinion publique qui permet de disposer de repères pour savoir ce qu'il convient de faire et ne pas faire.

40 Pour d'autres encore, l'éthique résulte de l'interaction sociale d'où émergent des règles qui définissent les critères d'efficacité et de légitimité. Ce sont ces règles implicites qu'il convient de mettre à jour lors du processus de délibération. À cet égard la conception de Padioleau ${ }^{19}$ est dans la continuité de l'approche positive des faits moraux de Durkheim.

41 Comme nous l'avons déjà montré, Padioleau ${ }^{20}$ décrit la possibilité pour l'acteur de se décentrer de sa pratique en décodant les règles qui structurent le jeu social. Le décideur peut ainsi agir avec une plus grande conscience par rapport aux règles réelles qui gouvernent l'action. Padioleau met donc en avant la valeur morale de la vérité.

42 Le débat qu'ouvre Moussé ${ }^{21}$ au sujet de l'approche de Padioleau est intéressant. Moussé part de l'idée qu'il existe une diversité des contenus de l'éthique et une similitude des formes d'évolution de la conscience morale. Si l'on prend comme acquise l'idée d'un développement d'une maturation morale de la personne, ce qu'il reste à résoudre est l'existence d'un va et vient entre une conscience autonome et libre et l'exigence de l'adhésion de la conscience à des valeurs universelles.

43 Tout le problème alors est de s'accorder sur ce principe universel. Etudier l'éthique oblige l'auteur à poser des convictions concernant l'homme et son devenir. C'est sur cette base 
que, dans son dernier ouvrage, Moussé souligne la limite de l'approche de Padioleau. Le problème que pose Moussé est de déterminer jusqu'où l'objectivité recherchée par le sociologue est possible. Or le fait même de défendre une conception positive de l'éthique est un choix subjectif. En recherchant l'honnêteté dans ses descriptions, il opte pour une méthodologie de recherche en fonction d'un a priori éthique et non sociologique. « Il se veut $"$ positif au nom d'une valeur finalement subjective $»^{22}$. Il nous semble donc que tout modèle concernant l'éthique des affaires véhicule une certaine idée et un arrière fond moral plus ou moins explicite.

Nous proposons la schématisation suivante des différents statuts que l'on attribue à l'éthique des affaires :

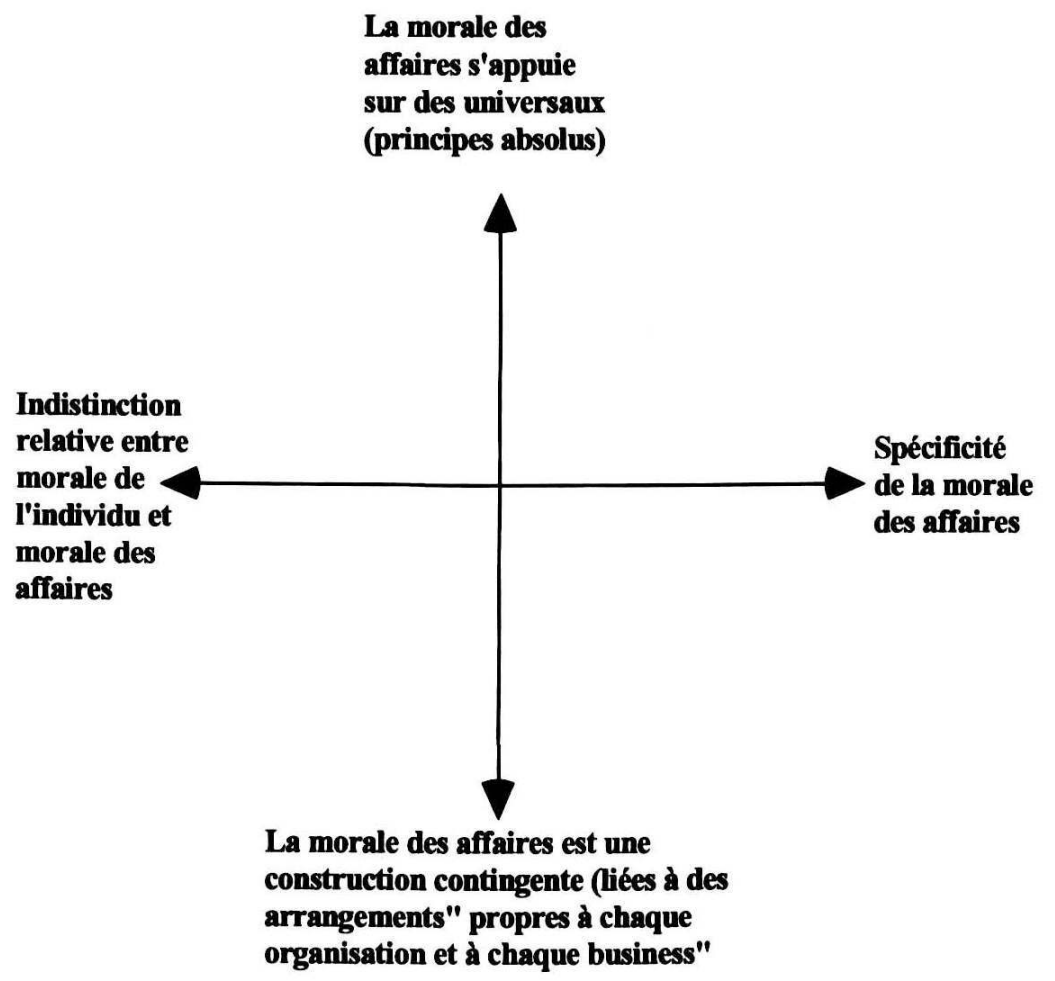


Tendance de ceux

qui défendent

l'existence d'une

éthique des affaires

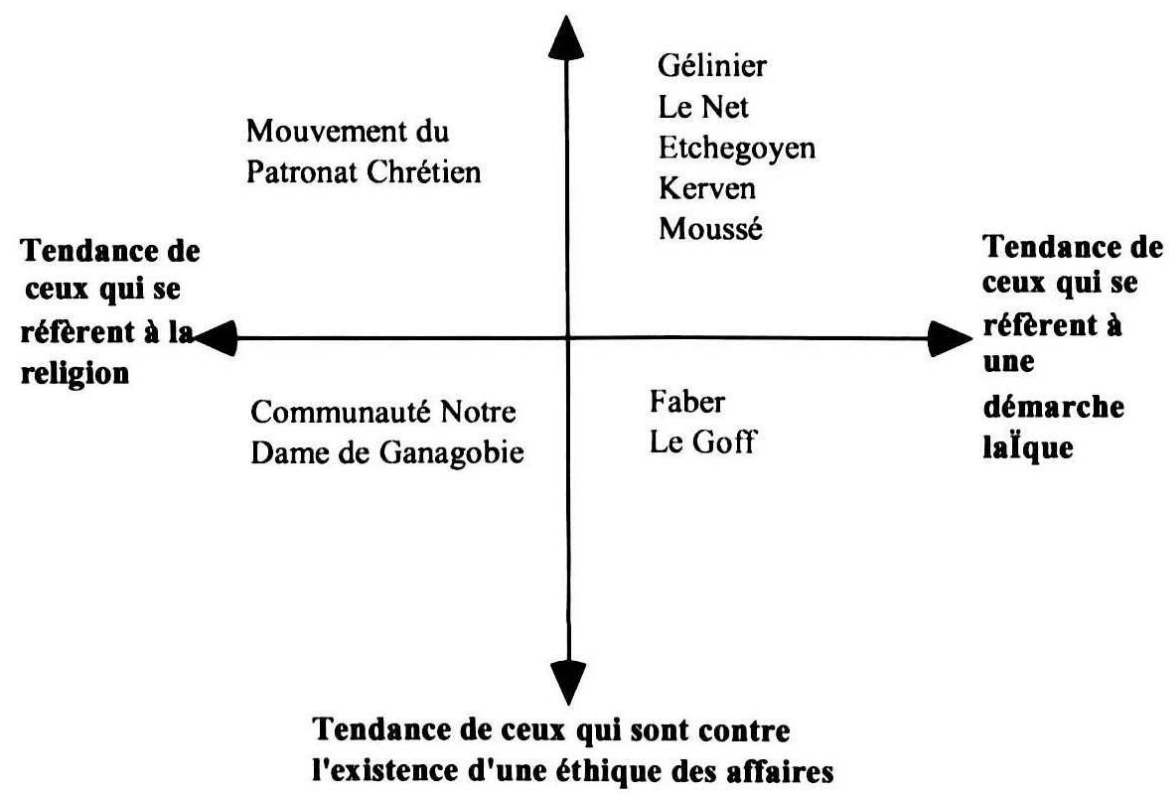

51 Selon nous, le problème essentiel à retenir est celui que pose Moussé. En effet, cet auteur considère que le dilemme fondamental de l'éthique concerne ce va et vient entre une conscience autonome et l'adhésion de la conscience à des valeurs morales.

Cela veut dire que les choix éthiques des managers face à une situation de travail posant problème résultent d'une réflexion plus ou moins complexe. Celle-ci prend en compte des valeurs sociales relatives, des principes plus généraux et une adhésion aux règles de l'entreprise. Nous venons de montrer que la normalisation de la démarche éthique peut prendre au moins trois formes. Ce qui est intéressant, au-delà des différences, c'est de constater que cette normalisation correspond à une volonté de structurer la relation entre une conscience autonome et des référentiels axiologiques. Au fond, étudier la manière dont le manager construit ses choix éthiques, ou plus précisément sa démarche éthique, revient à comprendre comment il construit l'interrelation entre sa conscience autonome (que nous comprenons comme sa capacité à donner sens à l'action) et les référentiels axiologiques collectifs. 


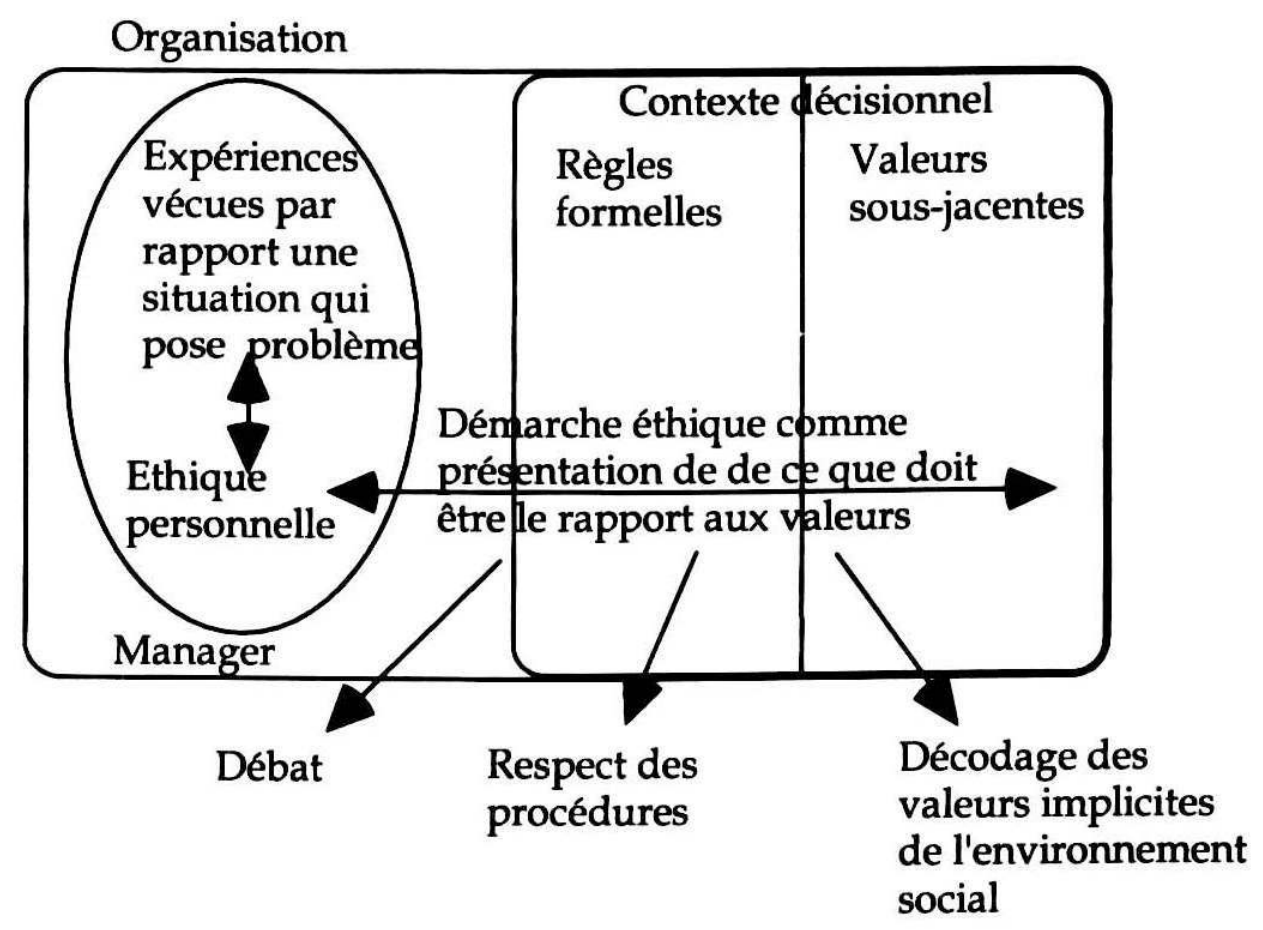

Si cette interrelation correspond à un mode de pensée rationnelle (au sens académique du terme) alors l'étude de la construction des démarches éthiques n'a plus de pertinence. Le seul problème qui se pose est celui de l'efficacité de la jonction des moyens aux fins. Ces dernières sont données une fois pour toutes et le manager doit s'y adapter. En d'autres termes, il n'y a plus lieu de parler d'éthique, mais de techniques, puisqu'on définit la technique comme une activité humaine qui consiste à utiliser des moyens pour parvenir à certaines fins ${ }^{24}$. Cependant la logique technico-économique n'est qu'une manière particulière de penser la relation aux finalités; elle correspond à un éclairage spécifique de la réalité : celui qui fait abstraction du débat sur le sens. Comme le souligne Orsoni ${ }^{25}$, les sciences de gestion se confrontent à un paradoxe intéressant: "la gestion n'est possible que parce que le monde est contingent. Dans un monde parfaitement rationnel, fabriqué comme une machine, où il serait établi que rien ne peut être autrement que ce qu'il est, il n'y aurait aucune place pour nous, les gestionnaires. Des scientifiques suffiraient, et leurs choix seraient automatiques. Au contraire, le fait qu'il y ait, dans le monde, des faits de hasards, inexplicables et imprévisibles, est une invitation, sans cesse renouvelée, à l'initiative de ceux qui sont appelés à gouverner nos modernes organisations. »

Nous allons maintenant développer cette dernière idée.

\section{Les limites de penser la démarche éthique du point de vue d'une activité réflexive rationnelle}

Il ne semble pas pertinent de penser la réflexion éthique selon une logique rationnelle de type théorique. Selon nous, la délibération associe de façon inextricable des composantes interpersonnelles et interpersonnelles, qui permettent à l'acteur de construire un engagement spécifique par rapport aux valeurs. 


\section{De la possibilité de délibérer sur les finalités dans un contexte d'entreprise}

Sur la base des éléments que nous venons d'évoquer, le processus de délibération se schématise comme suit ${ }^{26}$.

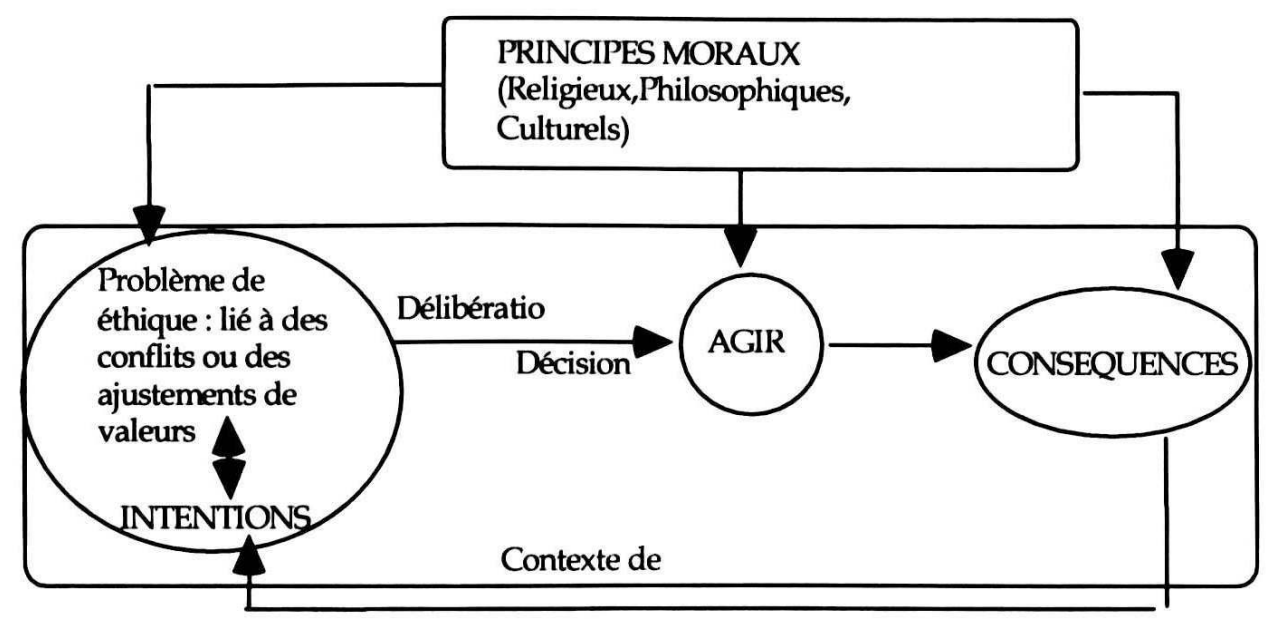

57 La délibération s'inscrit dans un contexte historiquement délimité. Elle prend en compte les enjeux d'une situation et les conséquences possibles des actions. En outre, la séquence, intention-délibération-décision-action, s'inscrit dans un substrat de valeurs morales, plus ou moins explicité.

Comme nous l'avons souligné, certaines approches de la délibération s'intéressent davantage aux principes moraux universels. D'autres accordent de l'importance aux règles structurant le contexte de l'action. On peut considérer que l'idéal moral du manager ainsi que les problématiques du contexte d'action influencent la manière dont le gestionnaire débat face à une situation qui pose problème.

Nous avons vu que certaines critiques portent sur le risque d'un cloisonnement de l'éthique des affaires dans une logique technico-économique, en délaissant des interactions souvent complexes qui président à la décision. Moussé ${ }^{27}$, dans son dernier ouvrage, insiste sur la nécessité d'ouvrir une démarche dialectique entre les principes moraux fondamentaux et les contingences des règles d'action. Il illustre ses propos en posant le problème de la rencontre difficile entre le philosophe et le gestionnaire.

"Aussi longtemps que la différence subsistera, c'est-à-dire que les décideurs n'intégreront pas la réflexion dans leurs décisions et que les philosophes ne feront pas l'analyse détaillée des conditions politiques, économiques, sociales et techniques de ces décisions, l'éthique des affaires restera dans le vague. Comme toutes les modes passées, elle cédera la place à une suivante ${ }^{28}$.

Cette critique nous permet de constater que l'appel à la raison n'est qu'un moment particulier du débat éthique. Son usage ne remplacera jamais la volonté qui s'exerce par l'attention mutuelle que nous nous portons les uns aux autres. Nous écoutons, nous entendons, nous sommes tendus les uns vers les autres, nous étirons notre esprit, bref, nous voulons. Ce n'est pas la philosophie qui est importante en elle même, mais le moyen qu'elle représente lorsque nous voulons mieux comprendre notre vécu. Comme le souligne Moussé, « la philosophie est indispensable à condition de ne pas la confondre, ni avec l'histoire, ni avec la critique de textes auxquelles on la réduit trop souvent. La 
question n'est pas ce que pensaient Kant ou Socrate, mais ce que nous pensons nousmêmes de la manière dont nous vivons, quitte pour cela à nous enrichir des idées kantiennes ou socratiques. $»^{29}$

61 Si l'on ôte la volonté et l'intentionnalité, les choix éthiques deviennent inintelligibles, même si les relations économiques restent compréhensibles. On perd en effet la capacité d'appréhender le rapport aux valeurs. Du simple fait qu'elles sont partagées, il est courant qu'elles le soient inégalement. Soit parce qu'il existe des niveaux de compréhension différents des valeurs, soit parce que certains prétendent les incarner mieux que d'autres. Soit enfin parce qu'il est possible de les hiérarchiser différemment. La liberté de la volonté est donc celle de lier ou de ne pas lier, d'accorder ou de désaccorder.

En outre l'usage de la raison est à distinguer de la nécessité de discerner les motivations profondes de l'action. Comme le soulignent Falise et Regnier ${ }^{30}$, «il faut se méfier à cet égard d'une vision fausse de l'éthique, qui ne prendrait en compte que les intentions et les mobiles de l'action. En réalité, intentions et mobiles sont souvent ambigus voire trompeurs. Et dans un monde complexe et difficile, seul un discernement attentif et rigoureux permet de mettre en œuvre les valeurs dont on se réclame. »

L'appel à la raison que sous-tend la notion de délibération est à relativiser. Nous voulons montrer que la réflexion n'est pas contradictoire avec l'action. Mais il semble important de préciser que cette réflexion se construit à partir de la manière dont le manager a "problématisé » le réel. Chacun construit son propre engagement par rapport aux valeurs. Weber ${ }^{31}$ qualifie celui-ci d'irrationnel pour montrer qu'il s'agit d'un acte de foi en lien avec la volonté de construire l'avenir.

64 Les finalités de la gestion ne sont pas construites une fois pour toutes. Elles sont mises en scène par l'action en fonction des réflexions sur soi-même et les événements. C'est parce que le responsable dispose de cette capacité à s'approprier et à s'accorder aux valeurs que nous pouvons nous interroger sur la manière dont il construit ses choix éthiques. Nous aurons l'occasion de revenir plus en détail sur cette proposition dans notre prochain chapitre. Mais nous voulons au préalable préciser ce que nous entendons par décision et la place de la délibération dans la décision.

\section{La question de la décision}

Ce qui distingue la délibération d'une décision immédiate, c'est la volonté pour le manager d'être conscient de la signification qu'il donne à son action.

Les décisions immédiates se justifient, soit parce que le problème a déjà été rencontré, soit parce que le décideur possède une expérience suffisante de la réalité ou qu'il n'estime pas que la situation présente un risque élevé. Il s'agit de l'action par habitude, avec une régulation apprise par l'expérience. Par contre si ces deux types de décisions sont porteurs de sens pour l'individu, rien n'indique qu'elles soient justes par rapport à une situation de gestion.

67 En ce qui concerne la prise de décision individuelle, de nombreuses recherches ont montré des heuristiques, c'est-à-dire les démarches mentales habituellement mises en œuvre dans les situations de décision. Ces recherches se sont surtout orientées vers une compréhension des mécanismes cognitifs. Elles ont notamment montré le caractère limité de la rationalité du décideur ${ }^{32}$ et l'impossibilité de rendre compte du processus de 
décision selon un schéma qui la fractionnerait en trois moments : préparation, décision, exécution ${ }^{33}$.

La décision est donc un processus qui s'inscrit dans un espace de multi-finalités, dans lequel les conflits et les contradictions, tant au niveau du champ social qu'au niveau de l'univers psychique, influencent les choix. Dans ce cas, la réflexion et l'action ne se situent plus dans une logique linéaire mais dans un processus.

Sans entrer dans le détail de ces différents champs de recherche, nous retenons que la décision est un phénomène abstrait qui repose sur des opérations mentales, non observables, directement effectuées de manière plus ou moins consciente. La délibération (ou réflexion), quant à elle, concerne les activités conscientes et volontaires qui permettent de penser l'action à venir et/ou de rationaliser celle effectuée.

La réflexion est donc à penser comme un processus par lequel le manager appréhende l'action pour parvenir au moment formel qui correspond au choix d'une option au profit d'une autre. Cette réflexion s'inscrit toujours dans un jeu d'aller-retour entre des convictions et des comportements réels. Il nous semble en effet que la réflexion ne peut émerger que sur la base de la manière dont la manager s'est représenté le réel. Or cette représentation est construite mais il ne s'agit pas d'une construction dans l'absolu. Elle est toujours plus ou moins en résonance avec une construction sociale de la réalité. Ce processus est fortement dépendant des aspects sociaux et psychiques.

71 En outre, étudier le comportement de la personne par rapport à la question éthique présente l'intérêt de montrer que ce questionnement intervient aussi au moment de la justification de l'action. Nous en concluons qu'une personne qui aurait agi par habitude a tendance à intégrer une réflexion morale au moment où elle doit justifier son action. La justification sera un moyen de la rendre acceptable vis à vis des tiers.

72 Face à une situation qui pose problème, la réflexion du manager est toujours partielle et partiale et ne s'inscrit nullement dans la logique d'une rationalité académique. Le manager est capable de penser l'action, mais sa réflexion est limitée par différents éléments. On peut supposer que la justification de l'action intégrera des valeurs qui dépassent les simples aspects technico-économiques. C'est d'ailleurs ce que montrent March et Oison $^{34}$ lorsqu'ils expliquent que l'acteur fonctionne selon une logique de justesse, c'est-à-dire qu'il agit en fonction des groupes sociaux auxquels il s'identifie. En effet, les acteurs s'efforcent d'établir des rapports de cohérence entre leur conduite et leurs rôles. L'action ne se déploie pas uniquement dans une perspective utilitariste. Elle est également orientée par la nécessité d'affirmation et de reproduction des identités.

73 Dans la réflexion interviennent conjointement une interprétation de la réalité, une sélection de l'information extérieure et une évaluation des enjeux de la situation en fonction du système de valeurs intériorisé par le manager. Nous reprendrons cette idée pour la développer dans le chapitre suivant.

Disons donc qu'il existe un lien rationnel entre la réflexion et la décision ${ }^{35}$. Cependant cette rationalité se heurte à 3 limites :

- La rationalité de l'acteur s'inscrit toujours dans des contextes d'action qui peuvent être interprétés de façons différentes.

76 - D'un individu ou d'un groupe à l'autre les marges de manœuvre réelles sont très variables. Cela dépend du statut et de l'influence relationnelle de la personne ou du groupe. 
77 - Les rationalités observables dépendent aussi des cadres de références de l'acteur (et de la relativité des modèles dont il dispose). Il peut prendre des décisions pour répondre à des motifs cachés et les présenter comme si elles étaient rationnelles.

Dans une logique intégrative, ces limites sont les éléments qui alimentent une réflexion. Ces restrictions composent ce que nous pourrions appeler une méta-délibération. Celle-ci est une décentration ${ }^{36}$ par rapport à la problématique du décideur. Elle prend en compte les mobiles et les motivations qui fondent la réflexion. Le décideur met alors en œuvre, ce que Louait appelle une logique intégrative, c'est-à-dire une capacité d'apprendre à apprendre.

En règle générale, nous savons que les acteurs délibèrent face à des situations de tension. Ce choix s'effectue à partir de leur connaissance limitée du bien et du mal, du juste et de l'injuste. L'acteur est cependant capable de développer cette connaissance. Mais cela implique qu'il se situe dans une logique intégrative par rapport à ses expériences et aux limites de ses modèles d'action. Nous pouvons alors considérer que ces limites résultent de l'ignorance des valeurs, des enjeux, des conditions et des effets de l'action. Les convictions de l'individu pourront aussi être des obstacles à la délibération.

En d'autres termes, nous pensons que le manager n'utilise pas seulement une logique de rationalité en finalité lorsqu'il construit ses décisions. Il semble hasardeux de prétendre que le gestionnaire aborde les choix éthiques selon une rationalité définie de manière théorique. C'est d'ailleurs l'idée qui est reprise par Hoaglund ${ }^{37}$. Cet auteur montre le hiatus existant entre la théorie et la pratique de l'éthique. Pour résoudre cette dissonance il est nécessaire d'améliorer notre compréhension de l'homme en tant qu'agent moral et de ne pas en limiter la définition à un être mono rationnel.

81 Pourtant, la tendance actuelle est d'appréhender le processus réflexif du responsable, en fonction d'un impératif intellectuel particulier, celui d'une rationalité en finalité ${ }^{38}$. C'està-dire qu'à l'intérieur d'un champ donné, le raisonnement est mené en fonction des moyens efficaces à mettre en œuvre pour atteindre les finalités particulières à ce champ.

Cette logique de pensée est fondée sur une représentation de l'individu comme objet. La personne est alors perçue comme un instrument, un rouage permettant l'accomplissement des objectifs. À cet égard, nous pouvons nous référer à la réflexion que menait Obzekhan sur le rapport aux finalités de l'action. Cette réflexion a été menée dans le cadre de la planification stratégique d'entreprise, mais son contenu nous semble encore d'actualité pour une analyse sur l'éthique des affaires. Pour cet auteur, réintégrer le rapport aux valeurs en gestion implique de changer nos représentations mentales de la réalité. «On admettra qu'il est difficile de conserver une représentation du monde fondée sur une définition particulière des faits alors que nous ne sommes plus sûr de ce qu'est exactement un fait $\|^{39}$.

83 Obzekhan, l'écueil principal d'une logique pensant l'homme de manière instrumentale est une cristallisation excessive des modes de pensée et d'agir selon une logique mécaniste. « Nous appréhendons la réalité - constellations d'objets, de problèmes et de solutions principalement sous la forme de dispositifs et de procédés mécanistes ${ }^{40}$.

Par exemple, la technologie est maintenant la composante centrale, peut-être même la composante essentielle de notre représentation du monde. En effet, on ne découvre pas l'avenir dans le sens d'une réalité préétablie qu'il suffirait d'éclairer à l'aide des instruments de mesure (principes moraux généraux et/ou données factuelles). On l'invente sur la base des décisions présentes qui deviennent des faits dans le futur. Le 
problème d'une telle conception du monde, qui pense pouvoir découvrir l'avenir, est qu'elle exclut du champ des possibles tout ce qui ne fait pas partie du faisable. Et puis le risque est aussi de sombrer dans le fatalisme, puisque ce qui doit arriver, arrivera.

«La sélection des fins doit avoir le pas sur l'évolution logique des moyens. Il s'ensuit donc, que de tels avenirs pourraient (et peut-être devraient) être imaginés comme radicalement différents de la réalité présente, et qu'ils devraient représenter des situations qui ne constituent pas de simples prolongements temporels de l'ici et du maintenant, et soit dégagés de la pesanteur de ce que nous pouvons simplement prédire $»^{41}$.

Dans cet avenir à inventer, le débat éthique fait partie intégrante de la réflexion, car la question qui se pose concerne l'avenir voulu. Ce qui implique qu'étudier la manière dont les managers construisent leur démarche éthique est possible car ils agissent et réagissent par rapport à la structuration axiologique de l'organisation.

\section{Conclusion}

Etudiée à un niveau organisationnel, l'éthique des affaires a pour fonction de définir le caractère et la conduite des collaborateurs en leur fixant des normes. Les dirigeants d'entreprise définissent ainsi les activités moralement bonnes. En ce sens, l'éthique organisationnelle est présentée comme une instance autonome. Elle offre des idéaux et des normes d'action, mais elle ne reflète pas nécessairement les réalités psychologiques ou les pratiques quotidiennes de l'organisation, ont une importance dans la manière dont les acteurs construisent leur identité.

Le problème de formalisation d'une éthique organisationnelle repose sur le fait que rien ne pourra jamais remplacer la capacité de l'acteur à arbitrer lui-même les situations qui lui posent des problèmes de conflits et/ou d'ajustement de valeurs. C'est pourquoi de nombreux auteurs préfèrent mettre l'accent sur la nécessité pour les managers de délibérer par eux-mêmes en intégrant une démarche éthique qui leur soit propre. Il s'agit alors de clarifier les éléments avec lesquels ceux-ci entrent en débat pour construire leurs choix éthiques.

Il apparait alors important de ne pas occulter le fait qu'en tant qu'entreprise normative l'éthique peut être séparée des modes habituels de construction des choix des membres de l'organisation. Or, si elle est trop éloignée de la manière dont les acteurs s'approprient leur action, elle prend la forme d'un discours idéologique. Notamment parce qu'elle ne porte pas simplement sur l'évaluation des interactions sociales, mais qu'elle véhicule aussi des normes d'évaluation personnelle et de respect de soi.

C'est pourquoi, il nous semble nécessaire de pas concevoir le processus réflexif avec une rationalité qui soit indépendante des composantes intrapersonnelles et interpersonnelles. Il s'agit donc d'éviter toute forme de rationalisme éthique. 


\section{BIBLIOGRAPHIE}

BERGMANN André, « Ethique et gestion », in Encyclopédie de gestion, Paris, Economica, 1989.

BRUSLERIE Hubert, (de la), (sous la direction), Ethique, déontologie et gestion de l'entreprise, Paris, Economica, 1992.

FALISE Michel, Régnier Jean, Repères pour une Ethique d'entreprise, Lille, Centre d'éthique contemporaine, 1992.

GELINIER Octave, L'éthique des affaires, Paris, Seuil 1993.

GELINIER Octave, Moral de l'entreprise et destin de la notion, Paris, Pion, 1965.

GRENN ROY M, « Business Ethics as a Postmodern Phenomenon », Business Ethics Quartely, the journal of the Society for Business Ethics, vol 3, n 3, july 1993.

HOAGLUND John, «Ethical Theory and Practice : Is There a Gap ? », Journal of Business Ethics 3, 1984.

JARISLOWSKY Sylvain, «L'éthique en finance », Gestion, mai 1991.

LOUART Pierre, « Des apories du décideur à l'expérience de l'action. Rôle des méta-modèles comme soutien de la gestion créative », Cahiers de la Recherche, IAE de Lille, 1990.

LOUART Pierre, Succès de l'intervention en gestion des ressources humaines, Paris, Liaisons, 1995.

MOUSSE Jean, Ethique et entreprises, éd. Vuibert, 1992.

MOUSSE Jean, Fondements d'une éthique professionnelle, éd. d'Organisation, 1989.

MOUSSE Jean, « Le chemin de l'éthique », Revue Française de Gestion, Mars-Avril-Mai 1992.

NANTEL John A., WEEKS W.A., " L'éthique en marketing : d'une approche utilitariste à une approche déontologique », Gestion mai 1991.

ORSONI Jaques, «L'enseignant de gestion face à la morale d'entreprise », Revue Française de Gestion, Juillet-Août 1989.

ORSONI Jaques, « Thalès manger », Revue Française de Gestion, Mars, Avril, Mai, 1994.

PADIOLEAU Jean-Gustave, « L'éthique est-elle un outil de gestion? », Revue Française de Gestion, juillet-août 1989.

PERROT Etienne, « Une interrogation sans fin », Projet, Hiver 1990-1991, n² 24.

PESQUEUX Yvon, RAMANANTSOA Bernard, « La situation de l'éthique des affaires en France », Ethiques des affaires, $\mathrm{n}^{\circ}$ 1, janvier 1995.

SAIELLI Philippe, Contribution à l'étude de la démarche éthique en management, Thèse de Doctorat en gestion, Université de Lille 1, décembre 1996.

SFEZ Lucien, La décision, Paris, PUF, coll. Que sais-je, 1988.

SFEZ Lucien, Critique de la décision, Paris, Presse de la Fondation Nationale des Sciences Politiques, 1992.

SIMON Herbert. À, Administrative Behavior, N.Y., The Free Press, 1976. 
STARK Alan, « La morale en affaire n'est-elle qu'un vœu pieux? » Harvard l'Epansion, $\mathrm{n}^{\circ} 70$

Automne 1993.

THEVENET Maurice, Bibard Laurent, «GRH et éthique, quels points communs, quelles

différences ? " $3^{e}$ Congrès de l'AGRH, Lille 1992.

THIEL Marie-Jo, « La conscience, une instance dynamique pour la bioéthique », Ethique (La vie en question) $\mathrm{n}^{\circ} 15,1995 / 1$.

WEBER Max, Le savant et le politique, Paris, Christian Bourgois, col. 10/18, 1990.

\section{NOTES}

1. Saielli P., Contribution à l'étude de la démarche éthique en management, Thèse de Doctorat en gestion. Université de Lille 1, Décembre 1996.

2. Bergmann A., "Ethique et gestion, dans Encyclopédie de gestion, », Paris, Economica, 1989,

p. 1118.

3. Moussé J., « Ethique et entreprises », Paris, Vuibert, 1992.

4. Thévenet M., Bibard L., "GRH et éthique, quels points communs, quelles différences ?», 3 Congrès de l'AGRH, Lille 1992.

5. Voir à ce sujet Grenn R.M., «Business Ethics as a Postmodern Phenomenon, " Business Ethics Quartely, the journal of the Soceity for Business Ethics, vol. 3, n 3, july 1993.

6. Louart $\mathrm{P}$., "Succès de l'intervention en gestion des ressources humaines ", Paris, Liaisons, 1995.

7. Louart P., op.cit.,

8. Perrot E., « Une interrogation sans fin », Projet, Hiver 1990-91, $n^{\circ} 24$.

9. Orsoni J., «L'enseignant de gestion face à la morale d'entreprise », RFG, Juillet-Août 1989. pp. 1115 et 1116.

10. Moussé J., « Fondements d'une éthique professionnelle », Organisation, 1989.

11. Orsoni J., " Thalès manger ", RFG, Mars, Avril, Mai, 1994.

12. Falise M., Régnier J., "Repères pour une Ethique d'entreprise », Centre d'éthique contemporaine, 1992, pp. 51.

13. Louart P., « Des apories du décideur à l'expérience de l'action. Rôle des méta-modèles comme soutien de la gestion créative ", Cahiers de la Recherche, IAE de Lille, 1990.

14. Jarislowsky S., «L'éthique en finance », Gestion, mai 1991.

15. Gélénier O., « L'éthique des affaires », Seuil 1993.

16. Bruslerie H., (de la), (sous la direction), «Ethique, déontologie et gestion de l'entreprise », Économica, 1992.

17. Stark A., «La morale en affaire n'est-elle qu'un voeu pieux?» Harvard l'Epansion, $\mathrm{n}^{\circ} 70$ Automne 1993.

18. Nantel J.A., Weeks W.A., «L'éthique en marketing: d'une approche utilitariste à une approche déontologique. » Gestion, Mai 1991, p. 61.

19. Padioleau J.G., « L'éthique est-elle un outil de gestion? », RFG, juillet-août 1989.

20. Padioleau J.G, op. cit.,

21. Moussé J., « Éthique et entreprises », Vuibert, 1992.

22. Moussé J., op. cit., p. 54.

23. Pesqueux Y., Ramanantsoa B., «La situation de l'éthique des affaires en France ", Ethiques des affaires, $\mathrm{n}^{\circ}$ 1, Janvier 1995, p. 18.

24. Orsoni J., op.cit., pp. 31.

25. Orsoni J., op.cit., pp. 35. 
26. Ce schéma est inspiré de Thiel M.J., «La conscience, une instance dynamique pour la bioéthique », Ethique (La vie en question) n 15, 1995/1.

27. Moussé J., « Ethique et entreprises », Vuibert, 1993.

28. Moussé op. cit., p. 15.

29. Moussé J. op. cit., p. 67.

30. Falise M., Régnier J., op. cit., p. 95.

31. Weber M., «Le savant et le politique », Christian Bourgois, col. 10/18, 1990.

32. Simon, H.A., « Administrative Behavior », The Free Press, 1976.

33. Sfez L., «La décision », PUF, coll. Que sais-je, 1988.

34. March J. G., et Olson P., Rediscovering Institutions, The Free Press, 1990.

35. Louart P., Succès de l'intervention en gestion des ressources humaines, Liaisons, 1995.

36. Cette décentration est d'ailleurs l'aspect spécifique d'une maturation morale.

37. Hoaglund J., « Ethical Theory and Practice : Is There a Gap ? » Journal of Business Ethics 3 (1984) 201-205.

38. Lire à ce sujet la critique d'Ozbekhan concernant les impératifs intellectuels des sociétés modernes. Obzekhan H., "Vers une théorie générale de la planification ", Prospective et Politique (Colloque de Bellagio), OCDE, 1969.

39. Obzekhan H., op. cit., p. 48.

40. Obzekhan H., op. cit., p. 48.

41. Obzekhan H., op. cit., p. 86.

\section{RÉSUMÉS}

Cet article propose un état des lieux de la démarche éthique en gestion. Au-delà d'un discours normatif sur l'éthique et l'entreprise, il s'agit de mettre en évidence les raisons qui incitent les auteurs à intégrer la question de la délibération morale au cœur des préoccupations manageriales. Il s'agit également de repérer les différentes formes que revêt la démarche éthique en fonction des références philosophiques qui la sous-tendent. Le dernier objectif de cet article est de mettre en parallèle la question de la démarche éthique avec celle de la maturation morale.

The purpose of this article is to state the situation of the ethical management. We want show why the authors refer to ethic in the management decisions in a company. We also want to identify the different ways that an ethical decision can have according to the philosophical theories taken as reference. The last subject of this article is to compare the ethical decision with the moral development.

\section{AUTEUR}

\section{PHILIPPE SAIELLI}

Philippe Saielli est maître de conférences de gestion à l'université du littoral - Côte d'Opale. Il enseigne à l'IUT de Calais. 